\title{
PENETRATION OF SODIUM AND THIOCYANATE INTO THE POSTERIOR AND ANTERIOR CHAMBERS*
}

\author{
BY \\ V. EVERETT KINSEY AND ERIK PALM \\ From Kresge Eye Institute, Detroit, Michigan, and Örebro, Sweden
}

THE mechanism by which various constituents of the aqueous humour gain entrance to and exit from the posterior and anterior chambers has been studied extensively. In one such investigation (Kinsey and Palm, 1955), the rate of accumulation of the cation, sodium, and the anion, thiocyanate, was determined. Theoretical curves based on mathematical expressions for several possible modes of penetration into the two chambers were fitted to the experimental data, and values for the various coefficients of transfer were computed. Inferences were drawn concerning the modes of entrance to and exit from the two chambers. The results were in agreement with the idea that both ions enter the anterior chamber in a common manner, i.e. both by flow from the posterior chamber and diffusion (presumably through the iris blood vessels), but that they enter the posterior chamber by different means. The rate at which sodium accumulated in the posterior chamber was consistent with an hypothesis that this ion is transferred from the blood in a unidirectional manner, whereas that for thiocyanate suggested that a to-and-fro process from blood to posterior chamber is involved although no claim was made concerning the exact nature of the processes involved.

Davson (1955) disagreed with these conclusions, chiefly because the calculations for the rate of accumulation of thiocyanate did not take into account the amount of the ion bound by plasma proteins. He expressed the view that our conclusion concerning the differences in the mode of entrance of thiocyanate and sodium depends "largely on the different steadystate distributions between plasma and aqueous humour that are reached by the two ions". Since "the distribution ratio for the diffusible fraction of thiocyanate would become approximately unity were 25 per cent. of the thiocyanate to be bound", he concluded "tentatively, that there is probably little in the kinetic studies described by Kinsey and Palm (1955) to suggest that the modes of penetration of the two ions, sodium and thiocyanate, are not fundamentally the same".

* Received for publication March 7, 1958. 
In this paper, we wish to present further data on the question of binding of thiocyanate in the plasma, and to discuss Davson's criticisms concerning the implications of thiocyanate binding with respect to our conclusions regarding aqueous humour dynamics.

The question of the fraction of thiocyanate bound by plasma proteins was considered in our previous study. Analyses were made during the early part of this investigation to confirm a previously held belief by the authors and by others, including Davson, that approximately one-fifth of the thiocyanate in the plasma is bound to protein. The results seemed to support this idea and our initial calculations used to quantitate the observations were made on this basis.

As the investigation proceeded, we became less certain whether the difference in the concentration of thiocyanate found in plasma and ultrafiltrates of plasma represented bound thiocyanate or was due to an artefact caused by some other colour-producing compound. A small percentage of binding seemed to affect our values very little. So, without denying the existence of a certain binding, we concluded that no "appreciable binding" occurred and made no adjustment of plasma levels for non-diffusible thiocyanate in making calculations.

Because of Davson's contention that some thiocyanate is bound, we have performed additional studies involving dialysis experiments and ultrafiltration of plasma to which thiocyanate had been added. To determine thiocyanate, we used both the Aldrich method of analysis as recommended by Davson, and the ferric nitrate method which we had employed previously. Plasma was dialysed against saline containing $5 \mathrm{mg}$. per cent. thiocyanate, and plasma containing $5 \mathrm{mg}$. per cent. thiocyanate was ultrafiltered through cellophane under a pressure of 100 pounds per square inch. When employing the ferric nitrate method, separate analyses were performed on the plasma to determine the contribution of colour produced by some substance present in plasma which was not thiocyanate. An appropriate correction was made in computing the concentration of plasma to which thiocyanate had been added. The correction amounted to less than 5 per cent. of the total in every instance. Excellent agreement was obtained by the Aldrich and the ferric nitrate methods of analyses, but greater blank values are found with the latter method. This deficiency of the method, however, was more than offset by the troublesome fading of colour with the Aldrich method.

The results of six experiments using ultrafiltration and six using dialysis show that the amount of bound thiocyanate varied from 11 to 25 per cent. (average approximately 20 per cent.). This value is in agreement with earlier results reported by Kinsey and Grant (1942), and, of more concern here, agrees reasonably well with those reported by Davson. We conclude that Davson is correct in his contention that some thiocyanate is bound to the plasma protein. However, as will be shown, this does not invalidate 
our conclusion that the results are consistent with the hypothesis that the modes of entrance of sodium and thiocyanate into the posterior chamber are different. Actually, the flow rates computed on the basis of 20 per cent. binding are more consistent with those obtained by other methods of measurement.

The data for thiocyanate were recomputed allowing for 20 per cent. binding of the thiocyanate to plasma. The results are shown in Fig. 1.

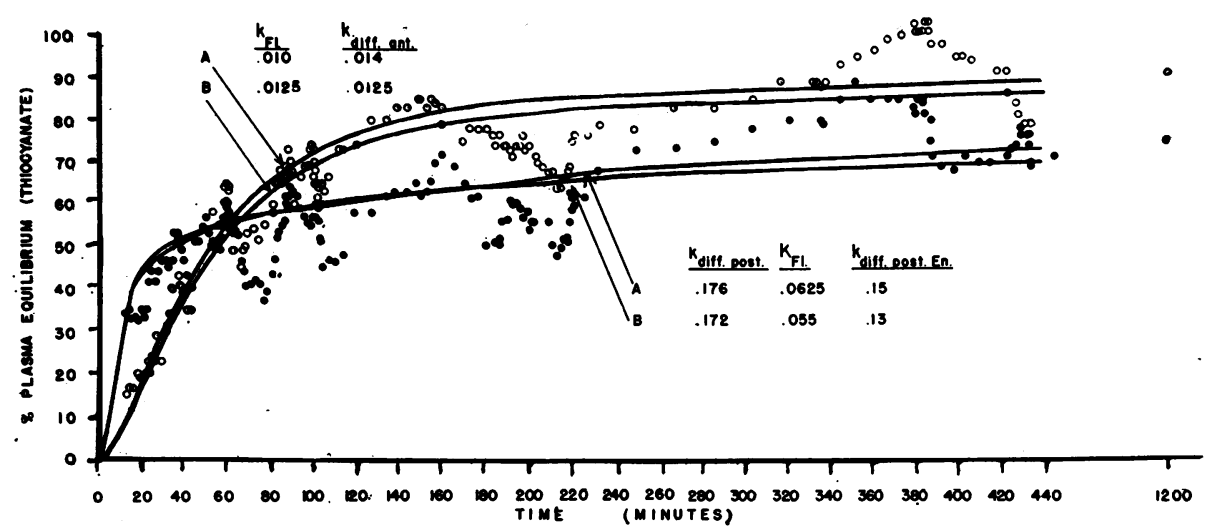

FIG. 1.-Relative concentrations of thiocyanate in the aqueous humour of the anterior and posterior chambers, where the concentration in the plasma once the level had become essentially constant was arbitrarily set at 100 per cent. The lines represent numerical integrations of the data using the values for the coefficients of transfer as indicated on the graph.

The open and filled circles represent sliding averages of the concentration of thiocyanate in the aqueous humour of the anterior and posterior chambers, respectively, relative to the plasma at various times after injection. The solid lines are curves resulting from numerical integrations of Equations 1 and 2 representing entrance into the posterior chamber by diffusion from the blood, and into the anterior chamber by flow from the posterior chamber and diffusion from the blood.

$\frac{\mathrm{dc}_{\mathrm{aq} .} \text { post. }}{\mathrm{dt}}=\mathrm{k}_{\text {diff. post. }}\left(\mathrm{C}_{\mathrm{pl} .}-\mathrm{C}_{\mathrm{aq} . \text { post. }}\right)-\mathrm{K}_{\text {flow }} \mathrm{C}_{\mathrm{aq} \text {. post. }}$

$$
-\mathrm{k}_{\text {diff. post. En }}\left(\mathrm{C}_{\mathrm{aq} \text {. post. }}-\mathrm{C}_{\mathrm{En} \text {. }}\right)
$$

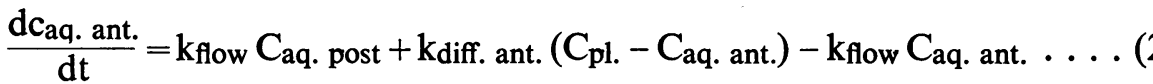

The values for the coefficients of transfer are shown on the charts. $\dagger$

Fig. 2 (opposite) shows the data obtained with $\mathrm{Na}^{24}$. In this instance, the lines were obtained from numerical integrations of Equation 3 for the data representing the posterior chamber and of Equation 2 for that representing the

* The symbols recently proposed by Duke-Elder and Maurice have not been employed in order to facilitate com-

parison with the previous paper. the same range were obtained $\left(k_{\text {flow }}=0.009\right.$ and $\left.k_{\text {diff. post. }}=0.012\right)$. 


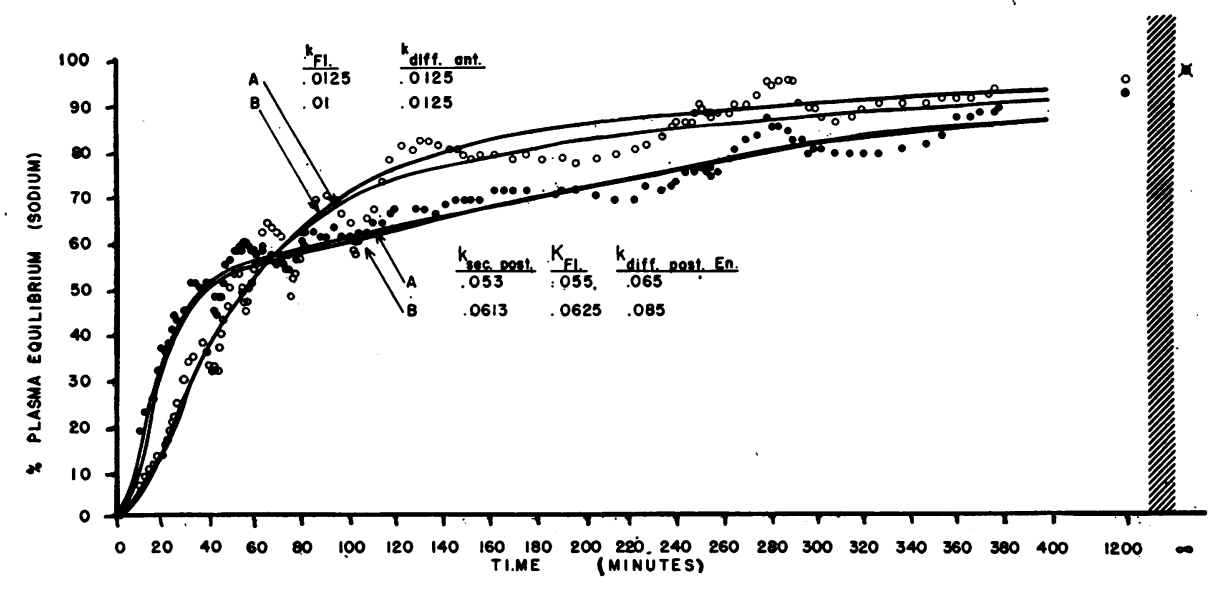

FIG. 2.-Relative concentrations of sodium in the aqueous humour of the anterior and posterior chambers, where the concentration in the plasma once the level had become essentially constant was arbitrarily set at $\mathbf{1 0 0}$ per cent. The lines represent numerical integrations of the data using the values for the coefficients of transfer as indicated on the graph.

anterior chamber. Equation 3 describes entrance into the posterior chamber by a unidirectional process:

$$
\frac{\mathrm{dc} \mathrm{aq}_{\mathrm{a} . \text { post. }}}{\mathrm{dt}}=\mathrm{k}_{\text {sec. post. }} \mathrm{C}_{\mathrm{pl} .}-\mathrm{K}_{\text {flow }} \mathrm{C}_{\mathrm{aq} \text {. post. }}-\mathrm{k}_{\text {diff. post. En. }}\left(\mathrm{C}_{\mathrm{aq} \text {. post. }}-\mathrm{C}_{\mathrm{En} .}\right)
$$

Relatively good fits to the data were obtained by numerically integrating the differential equations outlined above, representing the entrance of $\mathrm{Na}$ into the posterior chamber by secretion and of thiocyanate by diffusion. Moreover, a relatively good fit is obtained to both sets of data by numerically integrating the equation representing a dual means of entrance into the anterior chamber, i.e. by a combination of flow from the posterior chamber and diffusion from the blood. It will be observed that the coefficient of transfer for flow out of the anterior chamber (kflow) now lies in the range of $0.010-0.0125$, and that the present data, calculated with regard to a certain diffusibility, give rise to values for ( $\mathrm{k}_{\text {flow}}$ ) in better agreement than previously with that based on the use of paraaminohippurate.

The flow rate is somewhat lower than that which can be calculated from the data reported by Becker and Constant (1956), who determined the flow rate from experiments involving tonography and perfusion. They found the average flow rate to be $3.5 \mathrm{c} . \mathrm{mm}$./min. This value would correspond to a kflow of 0.014 , if it is assumed that the average volume of the anterior chamber of the rabbits employed was $250 \mathrm{c} . \mathrm{mm}$. Considering the fact that the degree of fit to our data is not highly dependent upon the numerical value of kflow, the limitations of accuracy of tonography in particular, and the possibility that the average weight of rabbits employed in the two 
different sets of experiments was different, the difference in flow rates, if any, is not considered to be significant.

It remains now to re-examine the question of whether equally good fits to the data can be obtained by calculating the rates of accumulation of the two ions in question in the posterior chamber on the assumption that they penetrate in the same manner. Various values of the coefficients of transfer for sodium into the posterior chamber were assumed and the numerical integrations were carried out on the basis of assumed entrance by diffusion. In no case could we obtain a fit to the experimental data at both ends of the curve. For instance, if it is assumed that $\mathrm{k}_{\text {diff. post. has a value of } 0 \cdot 10, \mathrm{~K}_{\text {flow }}}$ 0.0625 and $k_{\text {diff. post. En. }} 0.085$, a curve results which up to a time of 50 minutes corresponds well to the data. However, such assumptions lead to a steadystate value of approximately 60 per cent.-a value significantly below that found experimentally. If we substitute values for $k_{\text {diff. post. }}$ sufficiently large to give rise to steady-state ratios of near unity, the resulting initial rate of accumulation is much too rapid.

A reasonably good fit could be obtained for the thiocyanate data on the basis of assumed entrance by secretion using the values kflow 0.0625 , $\mathrm{k}_{\text {sec. }} 0.050$, and $\mathrm{k}_{\text {diff. post. }} \mathrm{En} .=0.043$.

The crux of the argument whether thiocyanate enters the posterior chamber by diffusion or secretion is not alone dependent upon the fit obtained to the data, but, as pointed out in the original paper, depends also on the degree of correspondence between the values computed for the relative ratio of the effective volume of the environment to posterior chamber using both sodium and thiocyanate data. This ratio may be computed from Equation 4.

$$
\frac{V_{\text {En. }}}{V_{\text {post. }}}=\frac{k_{\text {diff. post. En. }}}{k^{\prime} \text { diff. post. En. }}
$$

where $\mathbf{k}^{\prime}$ diff. post. En. is the coefficient of transfer for the filling of the lumped environment.

Since the value of $\mathbf{k}^{\prime}$ diff. post. En. for thiocyanate as determined experi-

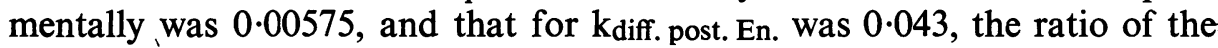
two coefficients is $7 \cdot 5$. The corresponding ratio for sodium, however, is $22(0.085 / 0.00384)$. The ratio for thiocyanate, assuming entrance is by

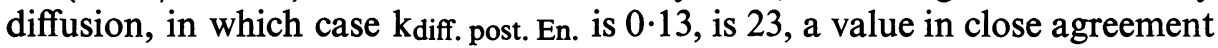
with that found for sodium.

It seems quite unlikely that the effective environment for thiocyanate is one-third that of sodium. In fact, Davson has suggested that the environment for thiocyanate is larger than that for sodium rather than smaller.

Thus, it is concluded that the data are, in fact, consistent with the hypothesis that sodium enters the posterior chamber unidirectionally which for lack of a better term may be called secretion, and thiocyanate enters by a two-way process, diffusion. 


\section{Summary}

The fraction of $\mathrm{SCN}$ bound to plasma proteins has been redetermined and in essential agreement with Davson (1955), found to represent approximately 20 per cent. of the total. Previously published data concerning the rate of accumulation of $\mathrm{SCN}$ into the posterior and anterior chambers have been corrected to allow for the fraction of SCN which is non-diffusible and theoretical curves based on mathematical expressions for several possible modes of penetration into the two chambers of the eye were fitted to the experimental data. Various coefficients of transfer were computed. Similar coefficients were recomputed for the rate of accumulation of $\mathrm{Na}$.

The data were found to be consistent with a flow rate of 1-1.25 per cent. per minute and with the hypothesis that $\mathrm{Na}$ enters the posterior chamber unidirectionally whereas SCN enters by a two-way diffusional process. They are also consistent with a hypothesis that both $\mathrm{Na}$ and SCN enter the anterior chamber by flow from the posterior chamber and diffusion from the blood across the iris-aqueous barrier. Both ions are believed to leave the anterior chamber by flow as well as by back diffusion through the iris.

\section{REFERENCES}

BeCKer, B., and Constant, M. A. (1956). A.M.A. Arch. Ophthal., 55, 305. Davson, H. (1955). Brit. J. Ophthal., 39, 681.

FriedenWALd, J. S., and BeCKER, B. (1956). Amer. J. Ophthal., 41, 383.

Kinsey, V. E., and GRANT, W. M. (1942). J. gen. Physiol., 26, 119. and PaLm, E. (1955). A.M.A. Arch. Ophthal., 53, 330. 